\title{
Emilio Modena und Robert Heim (Hrsg.): Unterwegs in der vaterlosen Gesellschaft. Zur Sozialpsychologie Alexander Mitscherlichs
}

\author{
(Psychosozial-Verlag, Giessen 2008) \\ Markus Weilenmann (Rüschlikon)
}

Pünktlich zum 100. Geburtstag von Alexander Mitscherlich haben Emilio Modena und Robert Heim einen wirklich lesenswerten Sammelband zur einst so gängigen Metapher der Vaterlosigkeit in unserer Zeit publiziert: Gemeinsam mit Beiträgen von Timo Hoyer, Hans-Joachim Busch, Hans-Jürgen Wirth, Angelika Ebrecht, Rolf Haubl und Johann August Schülein interessieren sich die Herausgeber für die Frage, ob heute von Vaterlosigkeit überhaupt gesprochen werden kann, ob es dieses Phänomen je gab. Weiter, welches Vaterbild für Alexander Mitscherlich denn wegleitend war, als er sich an die Niederschrift seines einstigen Bestsellers machte. Was wollte er damit erreichen? Wie lässt sich aus heutiger Sicht diese Metapher im noch jungen Nachkriegsdeutschland der 60er Jahre verorten? Und was sagen solche Metaphern über die Art und Weise aus, wie die noch junge Bundesrepublik Deutschland mit den Gräueln des Zweiten Weltkrieges umging? Gibt es Gemeinsamkeiten zwischen der von Mitscherlich diagnostizierten «Vaterlosigkeit» und der «Unfähigkeit zu Trauern»?

Die Autoren - alles Mitglieder des bestehenden Zirkels «Psychoanalyse - Gesellschaft - Kultur», der aus dem am Frankfurter Sigmund-Freud-Institut koordinierten «Arbeitskreis Politische Psychologie» hervorgegangen ist - tragen aus ganz unterschiedlichen Perspektiven Material zusammen, das die Diskussion zur Bedeutung der «vaterlosen Gesellschaft» neu anregt: Tim Hoyer führt den Leser in die Publikationsgeschichte des einstigen Bestsellers ein und dekonstruiert Mitscherlichs Erfolgsorientierung; Hans-Joachim Busch geht auf das Problem der Elternlosigkeit ein und legt dar, weshalb Mitscherlichs Werk auch als Beitrag zu einer Theorie der Bildung zu verstehen sei; Robert Heim sucht nach Verknüpfungen zwischen Lacan und Mitscherlich und präpariert dessen Vaterbild als Teil einer Anthropologie der Gesellschaft heraus, die auf einem hegelo-marxistischen Verständnis der Arbeitsgesellschaft beruht; Emilio Modena wendet sich anhand klinischer Fallstudien der Gegenwart zu und diskutiert Probleme heutiger Männeridentitäten; Hans-Jürgen Wirth setzt Mitscherlichs Ansatz in 
Bezug zur realen Vaterlosigkeit als Folge der Gräuel des 2. Weltkriegs und fragt nach Mitscherlichs eigener Art mit der Deutschen politischen Geschichte umzugehen. So zeigt sich, wie die «Unfähigkeit zu Trauern» gerade auch Mitscherlichs eigene, gesellschaftspolitische Analyse charakterisiert, indem seine Überlegungen durch eine fast schon penetrante Gegenwartsbezogenheit imponieren; Angelika Ebrecht zeigt auf, wie Mitscherlich die Funktion eines negativen Ichideals als Teil der Zwiespältigkeit moderner Vorbildfunktionen vernachlässigt. Dadurch bleibt seine Arbeit an außenorientierten Vorstellungen verhaftet und es gelingt ihm nicht, die innerpsychische Konfliktdynamik herauszuarbeiten; Rolf Haubl greift das Bild der Neidgesellschaft auf und nimmt sich Mitscherlichs problematischer Tendenz an, ganze Nationalstaaten und gesellschaftliche Gruppierungen einem (grob) verallgemeinernden Deutungsschema zu unterwerfen, ohne auch nur über einen annähernd hinreichenden Datenkörper zu verfügen und Johann August Schülein fragt abschließend, was aus Mitscherlichs Bestseller der «Vaterlosen Gesellschaft» heute geworden ist.

Gemeinsam ist diesem facettenreichen Zugang aber auch eine eher handzahme Auseinandersetzung mit Mitscherlichs Art, Sozialpsychologie zu betreiben und zu interpretieren. So stehen Mitscherlichs weit reichende Verallgemeinerungen in einem krassen Missverhältnis zu den empirischen Daten, die er präsentiert. Methodisch bleiben seine Arbeiten hinter dem sozialwissenschaftlichen Standard auch seiner Zeit zurück - erinnert sei beispielsweise an die vorbildlichen Arbeiten Bruno Betteilheims zur Massenpsychologie (1943) oder an Elias Canettis Masse und Macht (1960). Es mag darum auch kaum überraschen, dass seine Beiträge eher dem populärwissenschaftlichen Spektrum zugeordnet wurden und bis heute seine Thesen zu keiner weiter gehenden wissenschaftlichen Debatte führten. Ich hätte mir daher noch ein Methodenkapitel gewünscht, das Kernprobleme zur Entwicklung sozialpsychologischer Deutungsmuster kritisch ausleuchtet und die verbreitete Neigung der Autoren, Mitscherlichs verallgemeinernde Überlegungen gegen alle möglichen Einwände in Schutz zu nehmen, durch eine kompromisslosere Auseinandersetzung ersetzt. Dazu zähle ich etwa die Schwierigkeiten, die entstehen, wenn statistische Daten großer gesellschaftlicher Einheiten mit Aussagen zum Innenleben der Betroffenen verschränkt werden oder wenn von psychopathologischen Einzelfällen aus der eigenen Beratungspraxis auf gesellschaftliche Gesamtkonstellationen geschlossen werden soll. Darf man das? Welche Entscheidungsmaßstäbe werden solchem Tun unterlegt? Gibt es eine tatsächliche Datenkonvergenz oder wird sie einfach unterstellt? Assoziative Kreuz- und Querverbindungen mögen stets anregend sein, aber wenn sozialpsychologische 
Aussagen einen weiter gehenden Stellenwert beanspruchen wollen, genügt es eben nicht (mehr), allein auf die (imaginative) Kraft der Metapherbildung zu setzen.

Gefragt ist vielmehr eine auch methodisch überzeugendeAuseinandersetzung mit einem äußerst widersprüchlichen und heterogenen Datenkörper, die auch dem rauen Wissenschaftsalltag standzuhalten vermag.

Dies gilt umso mehr, als in verschiedenen Kapiteln auf interessante und auch äußerst wichtige Zusammenhänge hingewiesen wird, wie etwa auf das schon fast penetrante Gegenwartsverständnis einer genozidären Gesellschaft und die offenkundige Unfähigkeit auch Mitscherlichs, über die tatsächliche Vaterlosigkeit als Folge des Zweiten Weltkrieges eine öffentliche Debatte zu lancieren; oder auf den Zerfall eines autoritätsbezogenen Vaterbildes, das auf eine damals verbreitete Ablehnung gegenüber einer sich zunehmend pluralistisch strukturierenden Gesellschaft schließen lässt (Stichwort: «Die Unwirtlichkeit unserer Städte»); oder auf die zunehmende Feminisierung des Vaterbildes, eine Entwicklung die mehr schlecht als recht zur postulierten Vaterlosigkeit passt. Und so weiter.

Sie sehen, zum Diskutieren bietet dieses Buch reichlich Stoff.

\section{Literatur}

Bettelheim, Bruno (1943): Individual and Mass Behaviour in Extreme Situations.

In: Journal of Abnormal and Social Psychology, 38, 417-452.

Canetti, Elias (1960): Masse und Macht (1+2). Berlin: Claassen Verlag GmbH. 